\title{
Automated User Behavior Mapping Using Web Usage Mining
}

\author{
Dheeraj Ahuja ${ }^{1}$ | K C Tripathi ${ }^{1} \mid$ M L Sharma ${ }^{1}$ \\ ${ }^{1}$ Information Technology, Maharaja Agrasen Institute of Technology, Rohini, Delhi
}

To Cite this Article

Dheeraj Ahuja, K C Tripathi and M L Sharma, "Automated User Behavior Mapping Using Web Usage Mining", International Journal for Modern Trends in Science and Technology, 6(12): 257-261, 2020.

\section{Article Info}

Received on 10-November-2020, Revised on 02-December-2020, Accepted on 06-December-2020, Published on 11-December-2020.

\section{ABSTRACT}

Automated User Behavior Mapping is an application of web usage mining using which we can see the real-time behavior of end user visiting a particular web page automatically. The technologies used in this are socket programming for real-time communication between the server and the user accessing the website for collection of web log data and selenium web driver for automating the user behavior using web log files.

KEYWORDS: Web Usage Mining

\section{INTRODUCTION}

World Wide internet (WWW) is incredibly popular and interactive. It has become a crucial supply of data and services. The Internet is huge, various and dynamic. Extraction of fascinating data from internet has become common and as a result web mining has attracted lot of attention in recent time. The expansion of the internet (WWW) has showed the way of diverse client and server side tools development that mines the information resources to extract the intelligent knowledge. Web Mining is a process of extracting the knowledge and vital information from the World Wide Web. Web Mining[1] consists of three totally different classes:Web Content Mining, Web Structure Mining, and Web Usage Mining. The most important aim of web Usage Mining is to investigate the users' navigation patterns and their use of internet resources.

The primary focus of this paper is to map the user behavior using the concept of web usage mining and socket programming on the World Wide Web and automate the behavior using web drivers.

\section{LITERATURE SURVEY}

\section{A- Related Work}

Web usage mining [2] is the use of data mining strategies to find usage patterns from Web information, to comprehend and better serve the requirements of Web-based applications. Web usage mining comprises of three stages [3], specifically preprocessing, pattern discovery, and pattern analysis. This paper depicts every one of these stages in detail. Given its application potential, Web use mining has seen a fast expansion in interest, from both the research and practice communities. This paper provides an in depth taxonomy of the work in this space, including research efforts as well as commercial offerings.

Web Mining can be isolated in to three classes specifically Web Structure Mining (WSM), Web Content Mining (WCM) and Web Usage Mining (WUM). 
WSM tries to get the link structure of the hyperlinks at the inter-document level and generates a structural outline to look at information associated with the structure of a specific website.

WCM principally focuses on the structure of inner-document to seek out helpful information within the content of sites like free text within an online page, semi-structured knowledge like hypertext mark-up language code, pictures, and downloadable files.

WUM makes an attempt to find helpful information from the secondary information, particularly those contained in Web log files. Alternative sources may be browser logs, user profiles, user sessions, bookmarks, folders and scrolls. This information is obtained from the interactions of the users with the web. Effective web site management, making adaptive Websites, business and support services, personalization, and network traffic flow analysis expeditiously use WUM for higher performance. WUM focuses on the techniques that may predict user's navigation behavior.

Different web usage mining techniques are mentioned in [4] that may be used to extract patterns from web log files. Discovered patterns are used for pattern analysis that helps in understanding the user behaviors. As per [5], density-based clustering algorithm has been accustomed to discover navigation patterns. K-Nearest Neighbor algorithm with inverted index has been advised for economical prediction. Thus, many ways from data mining are utilized in the world of accustomed to usage analysis.

DOBBS [6] uses a browser add-on that permits researchers to log browsing behavior of on-line users, capture relevant and different window, session and browser events in anonymous and privacy-preserving manner and send those events to the server. In Dobbs, event is a unit of information. This paper describes all the logged events as well as window events, session events and browsing events. Window events includes events e.g. the opening and shutting of a browser window or tabs and changing within the state of browser window.

Passive browsing is that the time of idleness or inactivity throughout a user's browsing sessions. Parallel browsing is opening of multiple tabs inside one browser window and changing among them. Authors in [7] have analyzed in their study the impact of parallel and passive browsing on the calculation of user's time spent on web page.

Kosinski et al. [8] show that there's a mentally vital association between character, website and site categories. As indicated by this paper, outgoing clients' regularly visit sites known with Music and Social, whereas loners incline toward sites known with funnies, writing, and films. In addition, innovative and liberal are pulled in to journal, media, culture, crystal gazing, eBooks and expressive arts.

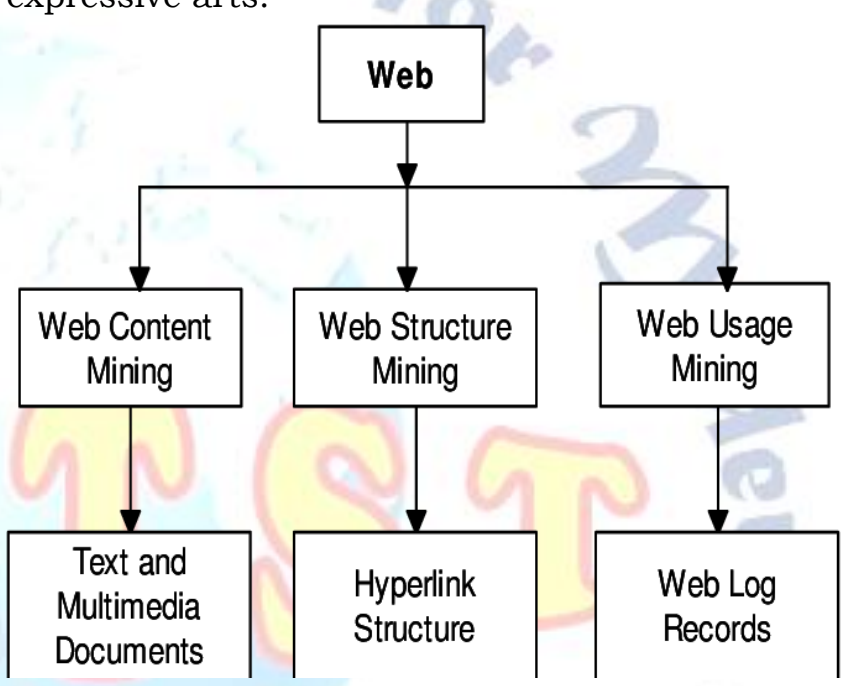

Figure 1: Web Mining and its types

\section{B-Related Applications}

\section{Personalization}

Customizing the Web experience for a client is the sacred goal of many Web-based applications, for example individualized showcasing for web based business (Making dynamic proposals to a Web client, in light of her/his parole notwithstanding utilization conduct is alluring to numerous applications). Web utilization mining is a great methodology for accomplishing this objective. A few tasks learns a client's inclinations by watching the page accesses for every user. A rundown of watchwords from pages that a client has invested a lot of energy seeing is gathered and introduced to the client. In view of input about the watchword list, suggestions for different pages inside the site are made.

\section{Updating in System Performance}

Performance of internet services may be an important drawback for user satisfaction. Web usage mining is a vital analysis space for locating internet traffic procedures, which may be 
accustomed amplify new policies for raising the web server concert $[9,10]$. Web caching technique transmission or account distributions, Load balancing, are the various application areas of web mining for performance upgrading.

\section{Site Restructuring}

The link association and content composition of any web site are two major factors in any internet site. the present trend in web mining tools go towards shorter navigation sequences, in support of that motive the accessibility of objective page in many internet domain must be improved [11]. Restructuring website topology of any internet field will achieve this. The restructuring task is performed with relevance the frequent patterns unconcerned at the end of web usage mining. Web usage data offers information in conjunction with the design of any web site with interests to user's activities [12]. To show page-stay moment offers the pages, that isn't attractive. Vendor of web site will re-establish these pages and observe the behavior of users on these pages. These two organizations techniques content and structure results in adaptive internet sites. The model given in changes web site organization with relevance usage patterns uncovered $[13,14]$.

\section{Supporting to the Design}

The Usability is most significant problems within the style and accomplishment of websites. The implications formed by web Usage Mining strategies will present ways for up the look of web functions. The utilize stereogram estimate the association and therefore the efficiency of websites from the clients view-point [15]. Web Usage mining strategies to propose acceptable variation for websites.

\section{Internet business}

Mining E-commerce intelligence from web usage information is considerably vital for web-based businesses. A good advantage from the utilization of web Usage Mining strategies will have client Relationship Management (CRM). Within this case, the aim is on business explicit issues such as: customer removal, customer attraction, cross sales, and customer retention [16] [17] [18].

\section{Methodology}

In this paper we present a way that will help you automated the mapping of user behavior on your webpages. It will use the concept of web usage mining for collection of web log data, socket programming for the communication between server and client and selenium web driver for the real-time automation of the web logs already generated at the server side.

\section{A)-Socket Pipeline Connection}

Socket programming is an approach of connecting 2 nodes on a network to speak with one another. One socket (node) listens on a specific port at an ip, whereas different socket reaches out to the opposite to make a connection. Server forms the listener socket whereas client reaches out to the server.

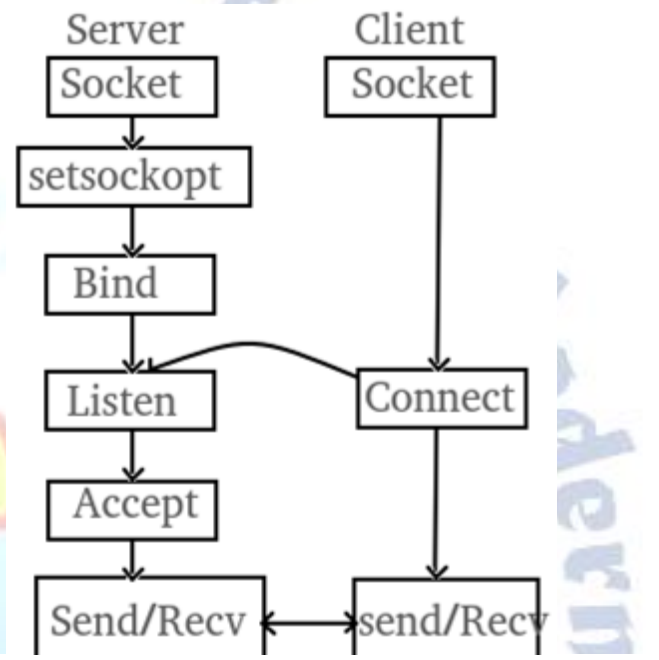

Figure2: State diagram for server and client model

As soon as the client connects from our server, we establish a socket pipeline connection between the server and the client and we capture few details about the user such as connection start time, user fingerprint containing various details such as browser, device, operating system, geo-ip, device-window-size, etc.

\section{B)-Collection of Web Log Data}

After collection of user's basic details we start storing user action that one performs on the web page. These include clicks, key press, and scrolling.

These actions are stored separately as one json-object. Each individual json action object consists of three fields - time, action and value.

Time field stores the value of the time difference between the previous action and the current action. Action field consist of one of the three action types i.e. click, key press and scroll. Value field consist of the value corresponding to these action types, click action stores the coordinates where the click action has been performed, key press event stores the key that has been pressed and scroll 
action stores the amount in pixels that the page has been scrolled.

Table I: Attributes of Web log data that helps in Automating User Behavior

\begin{tabular}{|c|c|}
\hline Attributes & Behavior \\
\hline Start Time & $\begin{array}{c}\text { Stores the start time of the } \\
\text { user session }\end{array}$ \\
\hline Browser & $\begin{array}{c}\text { Tells which browser has the } \\
\text { user used }\end{array}$ \\
\hline Os & $\begin{array}{c}\text { Tells which Operating System } \\
\text { has the user used }\end{array}$ \\
\hline Window Size & $\begin{array}{c}\text { Signifies the size of user } \\
\text { browser }\end{array}$ \\
\hline End Time & $\begin{array}{c}\text { Stores the start time of the } \\
\text { user session }\end{array}$ \\
\hline User Actions & $\begin{array}{l}\text { Records the time gap, user } \\
\text { action type and values of user } \\
\text { action }\end{array}$ \\
\hline
\end{tabular}

\section{C)-Automating the process using Selenium Web Driver}

Web Driver drives a browser natively, as a user would, either locally or on a remote machine using the Selenium server, marks a leap forward in terms of browser automation.

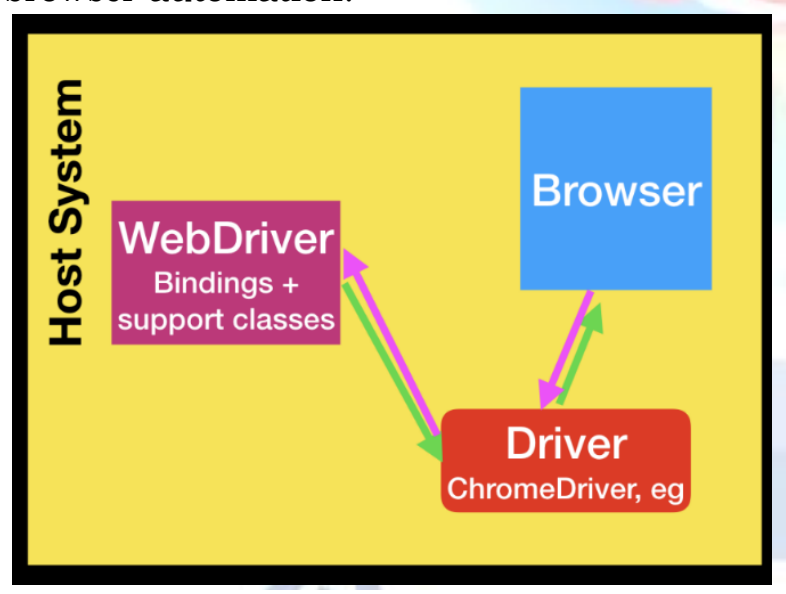

Figure 3: Components of Web Driver

The web log data file that has been created on the server and stores the user's details is now passed on to the selenium web driver script which starts reading the file line by line and mimics the action performed by them onto the web page on that web browser. Each action json object has three fields time, action and value so corresponding actions are executed after taking the pause (in millisecond) as stored in the time field.

\section{RESULTS}

In this paper we have implemented a tool using a blend of different concepts like web usage mining and technologies like socket programming and web drivers to automate the user behavior.

The architecture of the project developed is shown below:

Socket pipeline connection and collection of web log data

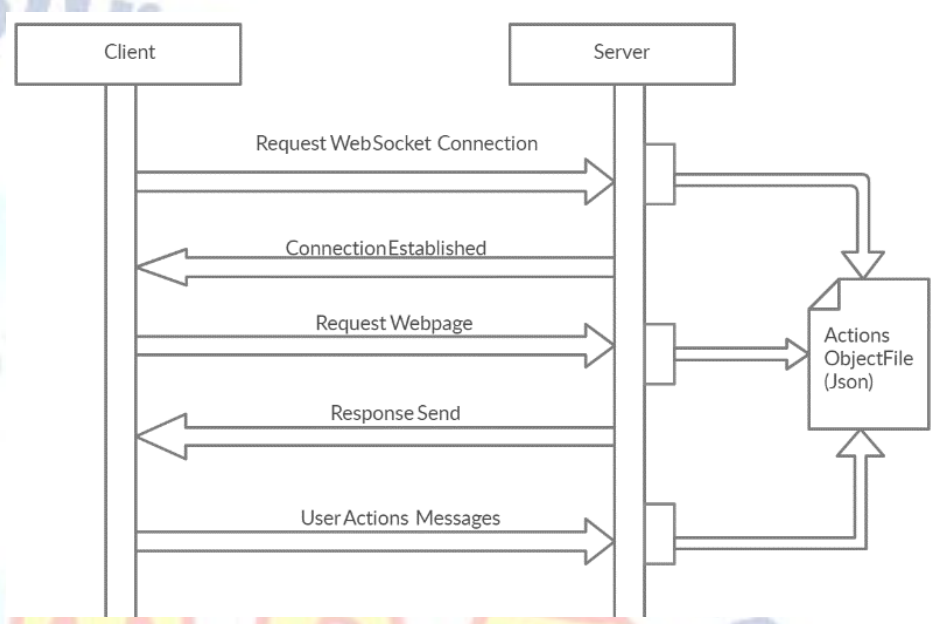

Fig4. The socket connection of the client and server and for the development of json object file has been shown here.

Automating the user behavior using web log data

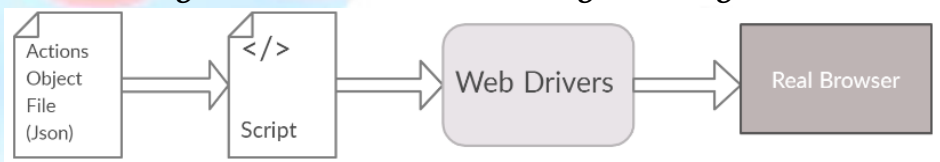

Fig5. The architecture for the automation process has been shown in the below diagram.

\section{CONCLUSION}

This paper has provided another application of web usage mining. With a large number of web applications being deployed daily, there is significant interest in analyzing Web usage data to better understand Web usage, and apply the knowledge to better serve users. We have used the concept of web usage mining for understanding the user behavior and automate it for getting insights related to user behavior.

\section{REFERENCES}

[1] J. Srivastava, R. Cooley, M. Deshpande, and P.-N. Tan, "Web usage mining: Discovery and applications of usage patterns from web data," ACM SIGKDD Explorations Newsletter, vol. 1, no. 2, pp. 12-23, 2000.

[2] Bhupendra Kumar Malviya,Jitendra Agrawal, "A Study on Web Usage Mining Theory and Applications" 2015 Fifth 
International Conference on Communication Systems and Network Technologies, pp. 935-939

[3] Farhana Seemi1, Hania Aslam2, Hamid Mukhtar3, Sana Khattak,"Browsing Behaviour Analysis using Data Mining "International Journal of Advanced Computer Science and Applications, Vol. 10, No. 2, 2019,pp.490-498

[4] M. Jafari, F. S. Sabzchi, and A. J. Irani, "Applying web usage mining techniques to design effective web recommendation systems: A case study," Advances in Computer Science: an International Journal, vol. 3, no. 2, pp. 78-90, 2014.

[5] P. Mehta, S. B. Jadhav, and R. Joshi, "Web usage mining for discovery and evaluation of online navigation pattern prediction," International Journal of Computer Applications, vol. 91, no. 4, 2014.

[6] C. von der Weth and M. Hauswirth, "Dobbs: Towards a comprehensive dataset to study the browsing behavior of online users," in Web Intelligence (WI) and Intelligent Agent Technologies (IAT), 2013 IEEE/WIC/ACM International Joint Conferences on, vol. $1 . \quad$ IEEE, 2013, pp. 51-56.

[7] "Analysing parallel and passive web browsing behavior and its effects on website metrics," arXiv preprint arXiv:1402.5255, 2014.

[8] M. Kosinski, D. Stillwell, P. Kohli, Y. Bachrach, and T. Graepel,"Personality and website choice," 2012

[9] Etminani, K.; Delui, A.R.; Yanehsari, N.R.; Rouhani, M.; "Web usage mining: Discovery of the users' navigational patterns using SOM", First International Conference on Networked Digital Technologies (NDT '09), Pp. 224 - 249, 2009.

[10] Pierrakos, D., et al, "Web Community Directories: A New Approach to Web Personalization", 1st European Web Mining Forum, pp. 113-129, 2003.

[11] Jing Wang, Ying Liu, Yong Shi, and Xingquan Zhu, Pushing Frequency Constraint to Utility Mining Model, ICCS 2007 Springer Verlag Berlin Heidelberg, Part III, LNCS 4489, 2007, 685-692.

[12] Kudelka, M.; Snasel, V.; Lehecka, O.; El-Qawasmeh, E.; "Semantic Analysis of Web Pages Using Web Patterns", IEEE/WIC/ACM International Conference on Web Intelligence, Pp. 329 - 333, 2006.

[13] Eirinaki,Magdalini,and Michalis Vazirgiannis, "Web mining for web personalization", ACM, vol. no. 1,pp 1-27,2003.ms and Knowledge Discovery (FSKD '08), Vol. 1, Pp. 52 - 56, 2008.

[14] Jain Pei, Jiawei Han, Behzad Mortazavi_asl and Hua Zhu, Mining Access Patterns Efficiently from Web Logs, PacificAsiaConference on Knowledge Discovery and Data Mining(PAKDD’00), Kyoto, Japan, 2000, 396-407.

[15] Chu-Hui Lee, Yu-lung Lo, Yu-Hsiang Fu, "A novel prediction model based on hierarchical characteristic of web site", Expert Systems with Applications 38, 2011.

[16] A. Anitha, "A New Web Usage Mining Approach for Next Page Access Prediction", International Journal of Computer Applications, Volume 8- No.11, October 2010

[17] Mehrdad Jalali, Norwati Mustapha, Md. Nasir Sulaiman, Ali Mamat, "WebPUM: A Web-based recommendation system to predict user future movements" Expert Systems with Applications 37, 2010.

[18] TrilokNathPandey, Ranjita Kumari Dash, Alaka Nanda Tripathy ,Barnali Sahu, "Merging Data Mining Techniques for Web Page Access Prediction: Integrating Markov Model with Clustering", IJCSI International Journal of Computer Science Issues, Vol. 9, Issue 6, No 1, November 2012.

\section{WEB RESOURCES}

[19] Socket IO https://socket.io/

[20] Selenium https://www.selenium.dev/

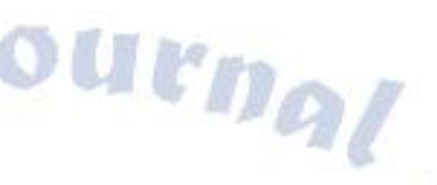

